\title{
Electronic Publication of VSI Articles One by One: A Paradigm Shift for Easy Accessibility, More Citations, and Survival of the Fitter
}

\author{
Suh Min Kim ${ }^{1}$, Soo Ho Kim², and Seung-Kee Min $^{3}$ \\ ${ }^{1}$ Department of Surgery, Chung-Ang University College of Medicine, Seoul, ${ }^{2}$ MEDrang lnc., Seoul, ${ }^{3}$ Division of Vascular Surgery, \\ Department of Surgery, Seoul National University College of Medicine, Seoul, Korea
}

\begin{abstract}
"Your beginnings will seem humble, so prosperous will your future be."

- The Bible -
\end{abstract}

The printed version of the June 2021 issue of the journal Vascular Specialist International (VSI) has just been published. As many readers may have noticed, VSl's publishing process has undergone a major evolution, so-called "oneby-one publication."

VSI is a peer-reviewed open-access international journal published every quarter at the end of March, June, September, and December in both electronic and printed versions. Although submission and peer review happen year-round through the online submission system, the journal being a quarterly publication means that authors of some accepted articles need to wait for up to three months before their work is published. To reduce this delay and increase accessibility, VSI adopted advanced online publication (ahead of print [AOP]) from May 2020, and free access to accepted articles is available on its website https://www.vsijournal. org/.

However, every three months, the editors have had to collect the articles to be published, align the contents, and allocate the page numbers, all of which put a huge burden on the editorial team. Recent advances in electronic publication and young users' preference for reading an article in the electronic rather than printed format thus motivated us to adapt. Based on the publishing company MEDrang Inc.'s proposal for one-by-one publication during the editors' workshop in February 2021, VSl's publisher and editors de- cided to take this big step forward. Each article is now published online as soon as it is accepted-the aim is to increase accessibility and possible citations. Major reputed international publishers such as Elsevier and Springer already have such a system in place. One-by-one publication has several advantages:

1) The quality of an article can be improved through intensive revision, and the editors can reduce the quarterly workload for meeting the deadline.

2) The manuscript editor and publishing company can distribute the workload.

3) The reader can easily access an article immediately after its acceptance.

4) Authors can derive greater satisfaction from the publication of their work right after its acceptance.

5) There is no need to accumulate articles in AOP.

6) There is no need to document a certificate of acceptance.

Furthermore, we have also decided to keep the printed version as is as per the preference of senior surgeons and collectors. Thus, the main changes are as follows:

\section{1) No sequential pagination of articles in an issue}

As you can see in the image of the contents page below, there are no page numbers for the articles (Fig. 1). The numbers 37:19 for the editorial mean 37th volume of VSI and 19th published article of the year. We grouped and arranged the articles according to their type, not the order of acceptance. However, in each issue, you can find every

Corresponding author: Seung-Kee Min, Division of Vascular Surgery, Seoul National University Hospital, 101 Daehak-ro, Jongno-gu, Seoul 03080, Korea

Tel: 82-2-2072-0297, Fax: 82-2-766-3975, E-mail: skminmd@snuh.org, https://orcid.org/0000-0002-1433-2562

This is an Open Access article distributed under the terms of the Creative Commons Attribution Non-Commercial License (http://creativecommons.org/licenses/by-nc/4.0) which permits unrestricted non-commercial use, distribution, and reproduction in any medium, provided the original work is properly cited. 


\section{Vascular Specialist International}

Vol. 37, No. 2, June 2021 pISSN 2288-7970 • elSSN 2288-7989

Editorial

37:19 Geriatric Vascular Surgery: Time to Build Optimal Resources for the Perioperative Care of Elderly and Fragile Vascular Patients

Seung-Kee Min

Review

37:14 Insights on Bridging Stent Grafts in Fenestrated and Branched Aortic Endografting William J. Yoon, Victor M. Rodriguez, Cheong Jun Lee

Original Articles

37:17 Gallium-67 Scan with Single Photon Emission Computed Tomography for the Evaluation and Monitoring of Infected Abdominal Aortic Aneurysms: A 10-Year Case Series

Hoi-Ming Kwok, Wing-Hang Luk, Lik-Fai Cheng, Nin-Yuan Pan, Ho-Fung Chan, Johnny Ka-Fai Ma

37:12 Association between Laterality and Location of Deep Vein Thrombosis of Lower Extremity and Pulmonary Embolism

Sangmin Gong, Eun Ji Lee, Jin Sung Kim, Hyangkyoung Kim, Minsu Noh, Hojong Park, Bong Won Park, Songsoo Yang, Sang Jun Park

Rapid communication

37:13 Distribution of Peripheral Arterial Disease in Patients Undergoing Endovascular Revascularization for Chronic Limb Threatening lschaemia: Insights from the Vascular Quality Initiative in Singapore

Shereen X. Y. Soon, Ankur Patel, Tze Tec Chong, Charyl J. Q. Yap, Hsien Ts'ung Tay, Kiang Hiong Tay, Chandramohan Sivanathan, Tjun Y. Tang

Case Reports

37:16 Do Not Tunnel the Small Bowel during the Tunneling of a Femoro-Femoral Bypass Chris Tae Young Chung, Sangil Min, Seung-Kee Min

37:15 Adventitial Cystic Disease of the Superficial Femoral Vein without a Joint Connection: A Case Report

Young Sup Yoo, Yoo Duk Choi, Ho Kyun Lee

37:18 Iliofemoral Vein Stenting in a Patient with Pelvic Metastasis Sina Zarrintan, Negin Yavari, Niki Tadayon, Fuad Majidi, Seyed Masoud Hosseini, Hamidreza Haghighatkhah, Ehsan Parvas, Seyed Moahammad Reza Kalantar-Motamedi

Fig. 1. Table of contents in the recent June 2021 issue of Vascular Specialist International. article's pagination starting from 1, showing "Page 1 of 8 " in the bottom outer corner.

\section{2) Change in citation methods}

Previously, VSI followed the Vancouver style for citations. However, articles in this issue have no sequential pages, and thus, every article should be cited with its digital object identifier (DOI). The citation format for VSI authors is as follows:

- Journal article with page numbers

Min SK, Kim YH, Joh JH, Kang JM, Park UJ, Kim HK, et al. Diagnosis and treatment of lower extremity deep vein thrombosis: Korean Practice Guidelines. Vasc Specialist lnt 2016;32:77-104.

- Journal article (electronic publication without page numbers, including VSI articles published after April 1, 2021)

Min SK. Geriatric vascular surgery: time to build optimal resources for the perioperative care of elderly and fragile vascular patients. Vasc Specialist lnt 2021. https://doi.org/10.5758/vsi.213721

This change does not influence VSI's evaluation process. The important feature meeting the prerequisites of the Korea Citation Index $(\mathrm{KCl})$ and national electronic solution for system academic activities (e-NEST) evaluation is the article 
number of each year, not each issue.

Another significant change that VSI has implemented is the revamp of its website homepage-the previous one was made in 2013, and was dedicated to ensure easy access for authors and readers. However, young surgeons want a novel design that is fancy and dynamic, and the preference for images and video content is increasing. Therefore, we decided to redesign the homepage to make it more interesting and fun, similar to Daily Prophet, the magical newspaper in the Harry Porter series (Fig. 2). The left column lists the recent articles posted with key figures; the middle one has videos (lectures, educational clips, and author interviews) playing to capture the viewer's attention-every member of VSI is welcome to submit any video related to vascular specialists; and in the right column, the most popular articles are posted for easy access. In the top-left corner, one can check VSI's metrics; next to it is the "My Read" button, which redirects to one's previously read articles, providing direct access to the articles of one's interest.

All these changes are to enable VSI to survive as a premier journal for vascular specialists [1]. Its success depends on the quality of the articles submitted and published and the citation index. VSl's editors aim to do their best to improve the quality of articles, the online submission system, and online accessibility.

\section{CONFLICTS OF INTEREST}

Seung-Kee Min has been the editor-in-chief and Suh Min Kim has been the managing editor of Vasc Specialist Int since 2019. Soo Ho Kim has been the director of MEDrang, the company which provides manuscript editing and print-

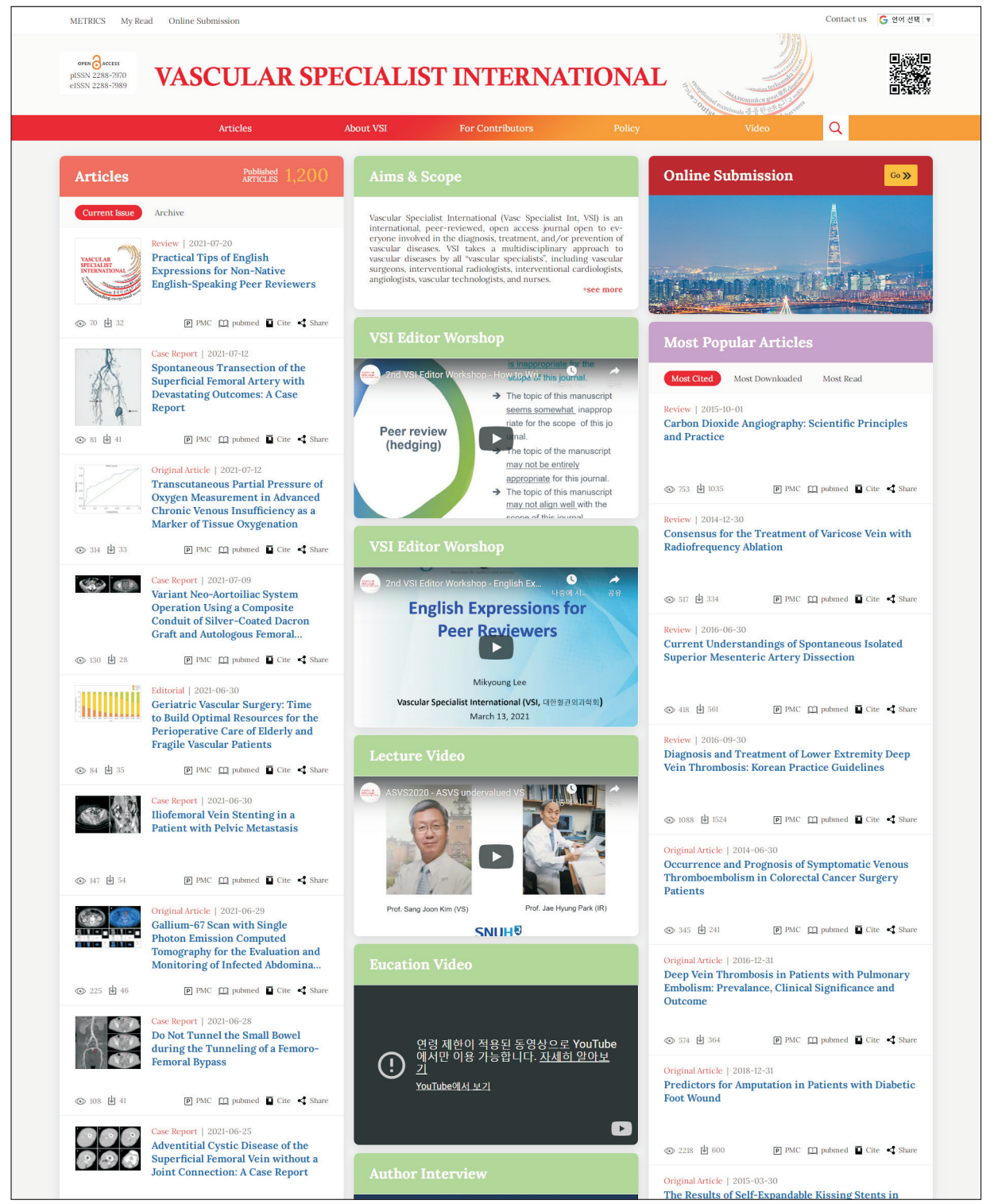

Fig. 2. Novel Vascular Specialist International website on https:// www.vsijournal.org/main.html. 
Kim et al.

ing service for Vasc Specialist Int.

\section{ORCID}

Suh Min Kim

https://orcid.org/0000-0003-3221-2190
Soo Ho Kim

https://orcid.org/0000-0002-9652-0929

Seung-Kee Min

https://orcid.org/0000-0002-1433-2562

\section{REFERENCES}

1) Min SK. Survival of the FITTER: moving forward together for a premier journal in vascular surgery. Vasc Specialist lnt 2019;35:1-3. 